\title{
Research on the Linkage Mechanism between Essential Medicine List and Healthcare Insurance List
}

\author{
Linning Chen ${ }^{1}$, Jinxi Ding1*, Jiaming Li1, Xuefang Yao \\ ${ }^{1}$ School of International Pharmaceutical Business, China Pharmaceutical University, Nanjing, China \\ ${ }^{2}$ School of Health Economics and Management, Nanjing University of Chinese Medicine, Nanjing, China \\ Email: *13605152326@163.com
}

How to cite this paper: Chen, L.N., Ding, J.X., Li, J.M. and Yao, X.F. (2021) Research on the Linkage Mechanism between Essential Medicine List and Healthcare Insurance List. Pharmacology \& Pharmacy, 12, 247-254. https://doi.org/10.4236/pp.2021.1211021

Received: November 3, 2021

Accepted: November 27, 2021

Published: November 30, 2021

Copyright $\odot 2021$ by author(s) and Scientific Research Publishing Inc. This work is licensed under the Creative Commons Attribution International License (CC BY 4.0).

http://creativecommons.org/licenses/by/4.0/ (c) (i) Open Access

\begin{abstract}
Objective: To put forward suggestions to improve the linkage mechanism between China's essential medicine list and healthcare insurance medicine list. Methods: Comparative study of the organization setting, selection criteria, adjustment procedures, and reimbursement of essential medicine list and healthcare insurance medicine list, containing both the foreign experience and China's status quo. Results: When two lists exist at the same time, they are often managed separately abroad, setting more selection criteria for the essential medicine, and giving the essential medicine a higher payment ratio. The two lists in China are managed and adjusted separately, but lack of connection. As a result, some essential medicines cannot be reimbursed. Conclusion: The two lists' linkage mechanism needs to be improved. It is recommended to make it clear that essential medicines should be selected from the healthcare insurance medicine list, and enhance the consistency of medicine evaluation through mutually scientific evidence.
\end{abstract}

\section{Keywords}

Essential Medicine List, Healthcare Insurance Medicine List, Linkage Mechanism

\section{Introduction}

The essential medicine system is the core content of China's national drug policy, and it has played an important role in improving the availability of medicines and ensuring the use of medicines in primary medical institutions. Because China's essential medicine system does not have an independent financing sys- 
tem, the reimbursement of essential medicines mainly relies on the healthcare insurance fund. In the past, essential medicines were exactly included in the healthcare insurance medicine list.

In 2018, the newly established National Healthcare Security Administration unified the responsibilities related to healthcare insurance, and sorted out the healthcare insurance medicine lists released previously. After that, a small number of essential medicines are not covered by healthcare insurance. After the essential medicine list was adjusted in 2018, 11 essential medicines have not yet been included in the healthcare insurance list. So far, the Tangcao tablet is still out of the healthcare insurance list. The composite potassium hydrogen phosphate was in the 2017 healthcare insurance list but was removed in 2019 .

Essential medicines and healthcare insurance medicines are both important to safeguard people's health needs. Essential medicines provide the optimal reference for clinical treatment, while healthcare insurance medicines pay for patients. The functional positioning determines the two lists should connect with each other, not only in the medicine varieties, but also in the selection criteria, adjustment procedures, etc.

This article aims to analyze the existing forms and connection modes of the two lists in foreign countries, and then explore ways to link up the two lists in China.

\section{Foreign Experience on the Adjustment of the Two Lists}

Through searching literature and public information, 105 countries and regions' essential medicine list and healthcare insurance medicine list were queried and included in the statistics. There are 25 countries or regions only have essential medicine list, and 17 countries or regions only have healthcare insurance medicine list (healthcare insurance medicine list refers to the medicine reimbursement lists, such as medicine formulary, pharmaceutical benefits scheme and so on), 63 countries or regions both have essential medicine list and healthcare insurance medicine list (Figure 1).

In countries with two lists, essential medicine list is either used directly as healthcare insurance medicine list, or is selected from the healthcare insurance medicine list. This article mainly studies the linkage mechanism of two lists, so the following will analyze such countries' experience.

\subsection{Organization Setting}

Countries in the distribution of the management responsibilities of the two lists are different, but the management can be divided into two lists: unified management and separate management. The management methods of the two lists abroad can be divided into two types: unified management and separate management. The Croatian Medical Insurance Agency (Hrvatski zavod za zdravstveno osiguranje, HZZO) uniformly manages the two lists, and the Ministry of Health is only responsible for reviewing and signing reimbursement agreements. The 
adjustment period is 1.5 - 2 years [1]. Sweden's healthcare insurance medicine list is formulated and dynamically adjusted by The Dental and Pharmaceutical Benefits Agency (Tandvårds och läkemedelsförmånsverket, TLV), while essential medicine list is formulated by the provincial medicine committees [2]. In Thailand, essential medicine list is formulated by the Ministry of Public Health (MOPH), while the Ministry of Finance, the Ministry of Labor and the National Security Office provides essential medicines for free [3]. The Brazilian Health department is responsible for the selection of essential medicines, and the Ministry of Finance pay for medicines [4].

\subsection{Selection Criteria}

The main purpose in formulating healthcare insurance medicine lists is to ensure the medical needs of people and reduce the burden of medication. Therefore, the selection criteria considered include safety and effectiveness, clinical needs, economic efficiency, ethical standards, and fairness, etc. In order to promote the rational use of medicines in clinical, the selection criteria in terms of safety, effectiveness for essential medicines are more stringent, as shown in Table 1 .

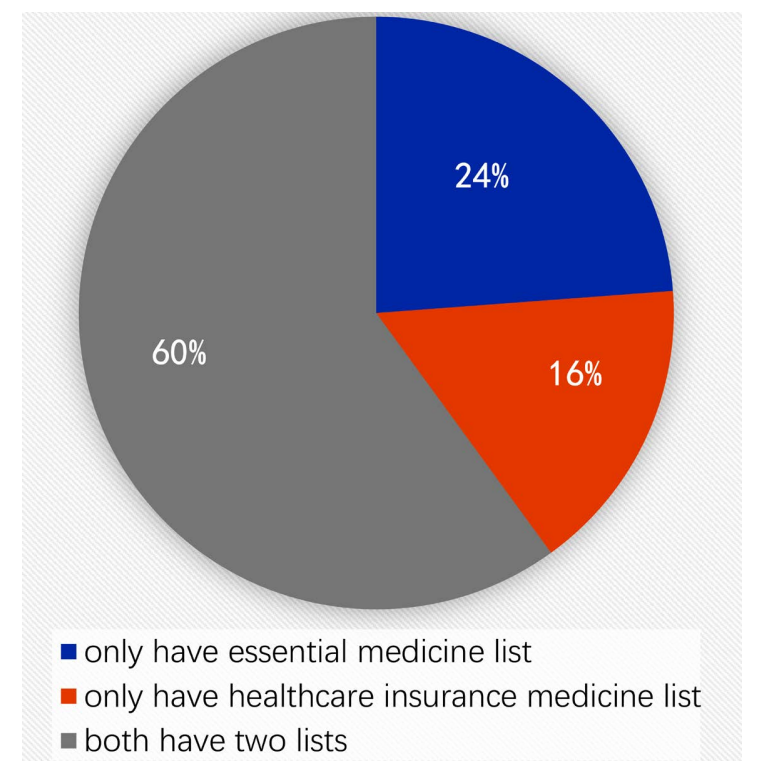

Figure 1. Status quo of the establishment of two lists abroad.

Table 1. Special selection criteria for the essential medicine list in some countries.

\begin{tabular}{cl}
\hline Country & Special selection criteria for essential medicines \\
\hline Croatia & Clinically necessary \\
Malaysia & Better safety and effectiveness, higher clinical needs, better cost-effectiveness \\
Sweden & The primary choice in clinical use, rational use \\
South & Used by primary health care or secondary/tertiary hospitals, meeting \\
Africa & essential medical needs, cost-benefit advantages \\
\hline
\end{tabular}




\subsection{Adjustment Procedures}

Corresponding to the organization setting, when the two lists are managed by the same department, the adjustments of the two lists are also integrated in the same procedures. For example, when Croatia formulates the healthcare insurance medicine list, at the same time, it classifies generic medicines and other clinically necessary medicines into the essential medicines list. But in most cases, the two lists are adjusted by different departments, and the adjustment procedure is relatively independent. Sweden's healthcare insurance medicine list is dynamically updated and adjusted. The admission decision is made within 180 days after the medicine manufacturer submitting the reimbursement application. The essential medicine list is in the charge of the provincial medicine committees and is selected from the healthcare insurance medicine list every year. South Africa's healthcare insurance medicine list consists of the essential medicine list and a supplementary list. The essential medicine list is adjusted by the National Essential Medicines List Committee (NEMLC) every two years. As for the medicines in supplementary list, they have higher price, and only can be prescribed in higher-level hospitals [5] (Figure 2).

\subsection{Reimbursement Policy}

Brazil, Thailand and other countries have used essential medicines as healthcare insurance medicine, their public health system provide essential medicines for free [4]. In countries with different healthcare insurance reimbursement policies, essential medicines usually have a higher reimbursement ratio. Croatian essential medicines are $100 \%$ reimbursed, and the reimbursement ratio for other healthcare insurance medicines is $10 \%-90 \%$. And there are no reimbursement

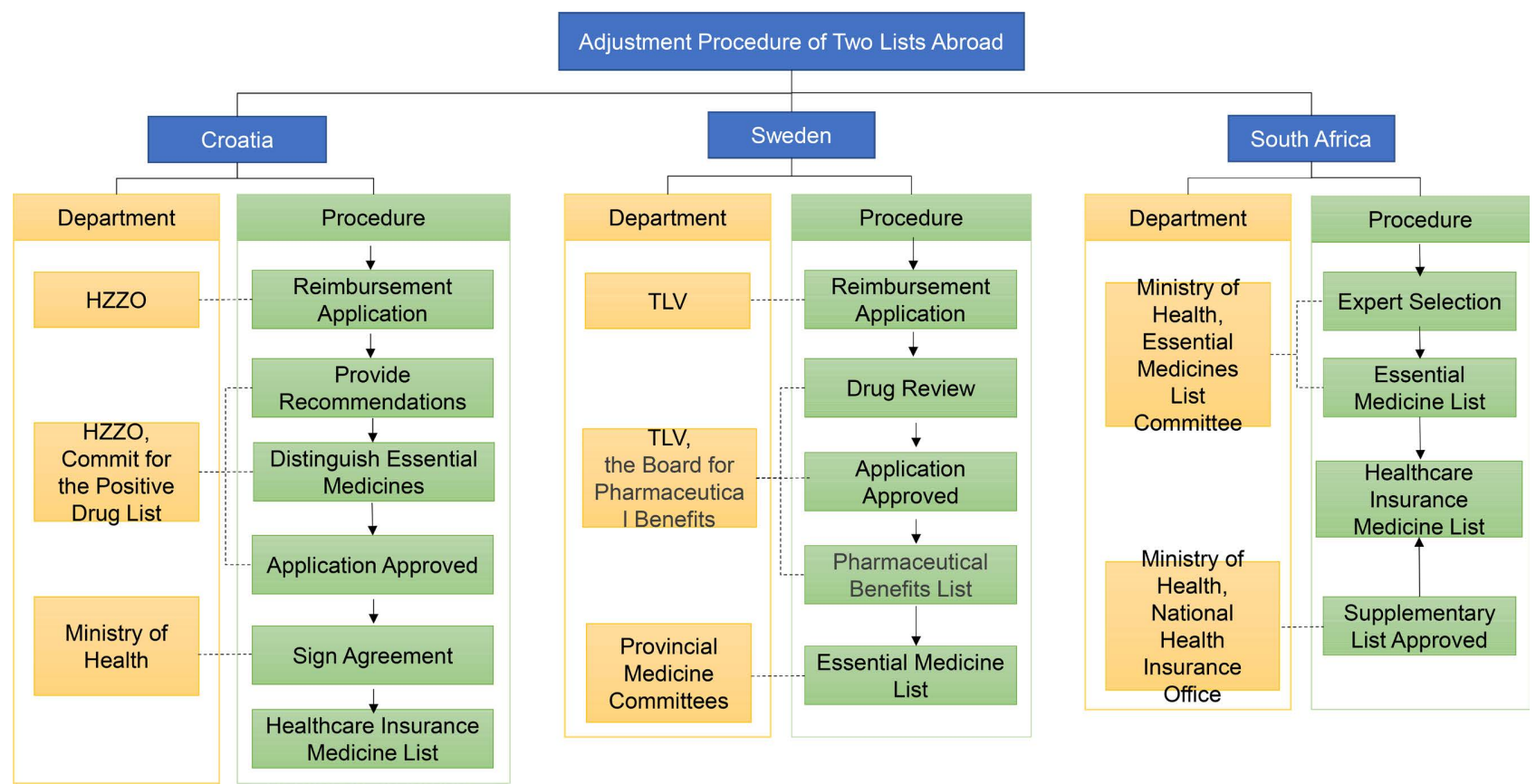

Figure 2. Flowchart for the adjustment of the two lists of some foreign countries. 
restrictions for specific populations or specific indications for essential medicines in Malaysia and South Africa [6]. Higher reimbursement benefits can guarantee the accessibility of essential medicines and guide clinical priority use, so as to reduce the burden of medicines.

The above experience shows that when the two lists exist at the same time, they are often managed separately. Meanwhile essential medicine list has more selection criteria, and higher payment protection.

\section{The Status Quo and Problems of the Linkage Mechanism of the Two Lists in China}

\subsection{Organization Setting}

At present, China will also manage the two lists separately. The essential medicine list is selected and managed by the Medicine Policy Department of the National Health Commission. In principle, it is adjusted every three years, but in fact it is longer than three years. The healthcare insurance medicine list is managed by the Medical Management Department of the National Healthcare Security Administration and adjusted dynamically every year.

\subsection{Selection Criteria}

One of the adjustment principles of China's healthcare insurance medicine list is to effectively guarantee the reasonable medicine needs of the insured persons, and insist on "essential protection". In the selecting procedure, the main consideration is the affordability of healthcare insurance fund and the insured persons. In summary, China's healthcare insurance medicines are safe, effective, clinically needed medicines with reasonable price.

Essential Medicines aim to guarantee essential medical needs, promote rational medicine use, so that limited medical resources can better meet the needs of the general public.

Therefore, China's essential medicines have more functions and more selection criteria, which are in line with international trends.

\subsection{Adjustment Procedures}

The adjustment procedure of essential medicine list and healthcare insurance medicine list is relatively independent. Two departments respectively design the selection methods and organize the experts participating in the review (Table 2).

Both lists are selected based on expert's review comments and scientific evidence. In 2020, the healthcare insurance list has begun to adopt the form of enterprise declaration, which can directly obtain first-hand data about medicine's efficacy and price [7]. The adjustment of the essential medicine list still relies more on expert's assessment results. The comprehensive clinical evaluation of medicines has become important evidence during the adjustment in 2018, but it still needs more improvement.

There was a problem with the linkage of the two lists in the adjustment 
Table 2. Adjustment process of the two lists in China.

\begin{tabular}{|c|c|c|}
\hline Procedure & $\begin{array}{l}\text { Healthcare insurance medicine } \\
\text { list }\end{array}$ & Essential medicine list \\
\hline Preparation & $\begin{array}{l}\text { Draw up a work plan, set up a } \\
\text { working organization and an } \\
\text { expert database }\end{array}$ & Set up an expert database \\
\hline Declaration & $\begin{array}{l}\text { Publish declaration guidelines, } \\
\text { receive, review and publicize } \\
\text { application material }\end{array}$ & - \\
\hline $\begin{array}{l}\text { Expert } \\
\text { review }\end{array}$ & $\begin{array}{l}\text { Expert review formed four lists: } \\
\text { "new include in", "direct remove", } \\
\text { "can remove", and "adjust the } \\
\text { payment policy" }\end{array}$ & $\begin{array}{l}\text { The consulting expert group selects } \\
\text { and forms a list of candidates, and the } \\
\text { review experts review and vote to form } \\
\text { the first draft }\end{array}$ \\
\hline Negotiated & $\begin{array}{l}\text { Part of the medicines with high } \\
\text { price negotiated and bid to } \\
\text { determine the healthcare insurance } \\
\text { payment standard }\end{array}$ & - \\
\hline $\begin{array}{l}\text { Solicit } \\
\text { opinions }\end{array}$ & - & $\begin{array}{l}\text { The first draft is solicited for opinions } \\
\text { from relevant departments, and } \\
\text { revised to form a second draft }\end{array}$ \\
\hline $\begin{array}{l}\text { Announce } \\
\text { results }\end{array}$ & Release a new list & $\begin{array}{l}\text { National Essential Medicine Working } \\
\text { Committee review and authorize the } \\
\text { release of new lists }\end{array}$ \\
\hline
\end{tabular}

procedure. Although both two lists need to include safe and effective drugs, using more repetitive expert resources, however, there is no consensus on the evaluation data of the two lists. In addition, the adjustment period is different, which means a drug will have to be reviewed twice to enter the two lists. It is easy to appear that essential medicines are not in the healthcare insurance list.

\subsection{Reimbursement Policy}

The two lists in China mostly overlap in variety. Compared with other healthcare insurance medicines, essential medicines have more reimbursement benefits. The current 2020 healthcare insurance medicine list include 2800 varieties. There are 683 kinds of essential medicines in the healthcare insurance medicine list, most of them can be reimbursed. Among them, about $67 \%$ of the varieties are in the class $\mathrm{A}$ list. About $4 \%$ of the varieties are both in the class $\mathrm{A}$ and class $B$ lists, which means that part of the indications of these medicines can be reimbursed according to the level of the class $\mathrm{A}$ list, and the others are reimbursed according to the level of class B list. (Medicine in class A list has higher reimbursement ratio) (Figure 3).

\section{Conclusions}

The two lists in China currently have an ambiguous and cohesive relationship. 


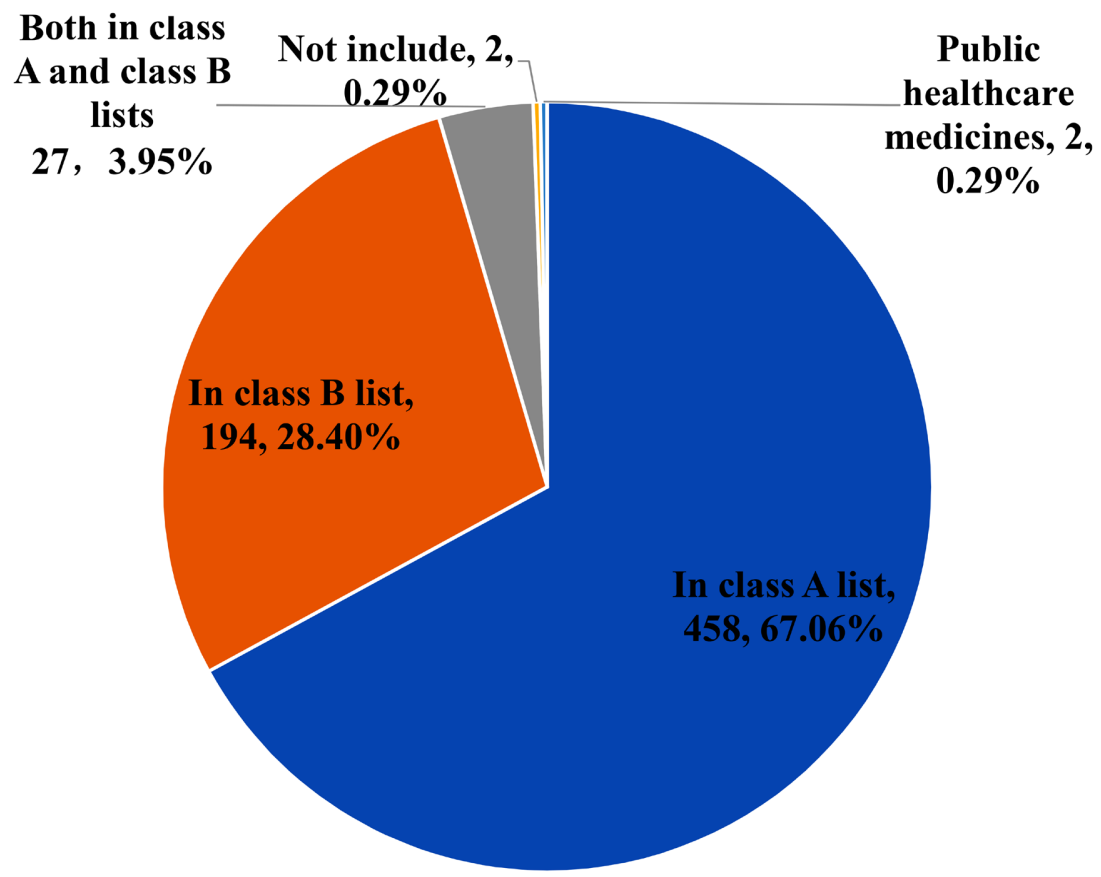

Figure 3. The status of essential medicines included in the healthcare insurance medicine list.

With independent adjustment procedures, the two lists lack a real specific linkage mechanism. Therefore, a small number of essential medicines cannot be reimbursed.

Learning from other countries' experience, selecting essential medicines can lead to the use of medicines from the healthcare insurance medicine list rational and logical. So far, the healthcare insurance list is dynamically adjusted every year, most of the newly marketed medicines are included in the healthcare insurance list in time, which means the healthcare insurance list is a good representative of the main clinical medicines. Meanwhile, guaranteeing the accessibility of essential medicines requires the support of the healthcare insurance fund. Essential medicines that cannot be reimbursed do not have good clinical guidance effect. Therefore, it is recommended to make it clear that essential medicines should be selected from the healthcare insurance medicine list. At the same time, the two lists can enhance the consistency of evaluation through mutual review or scientific evidence, and they can also reduce the waste of resources.

\section{Conflicts of Interest}

The authors declare no conflicts of interest regarding the publication of this paper.

\section{References}

[1] Culig, J., Antolic, S. and Szkultecka-Dbek, M. (2017) Drug Policy in Croatia. Value in Health Regional Issues, 13, 27-30. https://doi.org/10.1016/j.vhri.2017.07.005

[2] Pei, J., Lu, Y., Zhou, P., et al. (2018) The Development and Enlightenment of Swe- 
den's Essential Medicines List. Health Economics Research, 376, 49-51.

[3] Sun, J., Jiang, F. and Liu, Y.-L. (2017) The Successful Experiences of a Developing Country in Achieving Universal Access to Expensive Life-Saving Medicines and Its Implications to China. China Pharmacy, 28, 289-294.

[4] Santos-Pinto, C., Ventura, M., Pepe, V., et al. (2013) Essential Medicines and Technology Incorporation Following Novel Brazilian Public Health System Regulations. Cadernos de saúde públical Ministério da Saúde, Fundação Oswaldo Cruz, Escola Nacional de Saúde Pública, 29, 1056-1058. https://doi.org/10.1590/S0102-311X2013000600002

[5] South Africa Government (2003) National Health Act. National Health Insurance Policy, 8, 18-21.

[6] South Africa Government (2020) National Essential Medicines List Fourth Edition.

[7] National Healthcare Security and Administration (2020) Announcement on the Publication of the "2020 National Healthcare Security Medicine List Adjustment Work Plan”. http://www.nhsa.gov.cn/art/2020/8/17/art 37 3407.html 